\title{
CONSISTENCIA TERMODINÂMICA DE DADOS EXPERIMENTAIS DE EQUILÍBRIO DE FASES EM SISTEMAS BINÁRIOS
}

\author{
J.F. GUIMARÃES ${ }^{1}$, P.F. ARCE-CASTILLO ${ }^{1}$ \\ ${ }^{1}$ Universidade de São Paulo, Escola de Engenharia de Lorena, Depto de Engenharia Química \\ E-mail para contato: parce@usp.br
}

\begin{abstract}
RESUMO - Do ponto de vista da termodinâmica, nem todos os dados experimentais de equilíbrio líquido-vapor de sistemas binários e ternários que constam na literatura são corretos. $\mathrm{O}$ teste de consistência termodinâmica é o método mais eficiente para determinar a veracidade ou não destes dados. Este projeto visa aplicar o método de consistência termodinâmica a dados obtidos em experimentos de equilíbrio líquido-vapor, previamente selecionados e analisados, utilizando o modelo termodinâmico (equação cúbica de estado) de PengRobinson. Os dados experimentais foram submetidos aos testes de consistência termodinâmica fazendo-se uso de métodos numéricos e linguagens computacionais. Foram analisados dados experimentais de vários sistemas binários de equilíbrio líquido-vapor, dentre os quais encontrou-se dados considerados termodinamicamente consistentes e alguns inconsistentes. As análises comprovam que nem todos os dados experimentais de equilíbrio líquidovapor encontrados na literatura são confiáveis e que deve-se analisar a certeza de tais valores antes de fazer uso deles.
\end{abstract}

\section{INTRODUÇÃO}

Sabe-se que a equação de Gibbs-Duhem relaciona os coeficientes de atividade de todos os componentes de uma mistura. Se todos os dados necessários para o cálculo destes coeficientes estiverem disponíveis, deverão obedecer a esta equação; caso contrário, os dados não são verdadeiros ou houve erros graves na sua obtenção. No entanto, a conformidade dos dados com a equação não significa necessariamente que estes dados são corretos, pois é possível - mas não provável - que dados incorretos fortuitamente possam satisfazê-la. Infelizmente, na literatura encontram-se dados de equilíbrio de fases (equilíbrio líquidovapor) que não satisfazem a equação de Gibbs-Duhem e, portanto, são considerados incorretos sob a ótica da termodinâmica (McDermott e Ellis, 1965; Liebermann e Fried, 1972).

\section{CONSISTÊNCIA TERMODINÂMICA}

A análise de consistência termodinâmica pode ser feita utilizando-se diferentes métodos, os quais dependem do modelo termodinâmico. O objetivo principal é que o modelo seja capaz de ajustar exatamente os dados experimentais e possa ser utilizado para aplicar o teste de consistência termodinâmica baseado na equação de Gibbs-Duhem. $O$ teste de 
consistência termodinâmica pode ser aplicado a dados experimentais de equilíbrio para sistemas binários e sistemas ternários (Smith et al., 2007).

\subsection{Equação de Gibbs-Duhem e Propriedades Parciais Molares}

A equação de Gibbs-Duhem relaciona propriedades de mistura e propriedades parciais e pode ser escrita de maneira geral da seguinte forma:

$$
\left(\frac{\partial M}{\partial P}\right)_{T, x} \mathbb{d P}+\left(\frac{\partial M}{\partial T}\right)_{P, x} \mathbb{d} T-\sum_{i} x_{i} \mathbb{d} M_{i}=0
$$

Essa equação tem que ser satisfeita para todas as variações de pressão $(\mathrm{P})$, temperatura (T) e a propriedade parcial molar $\left({ }^{M}\right)$ causada por mudanças de estado em uma fase homogênea. Quando tem-se o caso particular, em que $\mathrm{P}$ e T são constantes, a Equação 1 simplifica-se na Equação 2:

$$
\sum x_{i} \mathbb{d} \bar{M}_{i}=0 \quad(\text { T e P contantes })
$$

Isto significa que se as propriedades parciais molares puderem ser calculadas, poderemos submeter dados termodinâmicos experimentais a um teste de consistência termodinâmica através da equação de Gibbs-Duhem.

Para o equilíbrio de fases líquido-vapor em sistemas binários a baixas pressões, pode ser feito um rearranjo na Equação 2. A Equação 3 mostra a expressão de Gibbs-Duhem em termos da propriedade molar parcial: o logaritmo natural dos coeficientes de atividade dos componentes no sistema binário:

$$
\int_{x i}^{x f} \ln \left(\frac{\gamma_{1}}{\gamma_{2}}\right) d x x_{1}=0
$$

Para sistemas binários a baixas pressões, pode-se calcular as propriedades parciais molares necessárias na utilização da equação de Gibbs-Duhem através da chamada abordagem gamma-phi (Smith et al., 2007), expressa na Equação 4:

$$
y_{i} \hat{\phi}^{V} P=x_{i} \gamma_{i} P_{i}^{\text {sat }} P O Y_{i} \phi_{i}^{\text {sat }}
$$

A abordagem gamma-phi $(\gamma-\phi)$ é um método muito eficiente de obtenção dos coeficientes de atividade para sistemas a baixas pressões. Nestas condições pode-se considerar o fator de Poynting (POY) e o coeficiente de fugacidade do componente i na saturação $\left(\phi^{\text {sat }}{ }_{i}\right)$ como valores próximos a 1,0. Sendo assim, a forma final da abordagem será a Equação 5:

$$
y_{i} \hat{\phi}^{V} P=x_{i} \gamma_{i} P_{i}^{\text {sat }}
$$

Fazendo-se uso da Equação 5, pode-se calcular os coeficientes de atividade dos componentes da mistura binária para cada valor de fração molar. Assim, será possível utilizar a equação de Gibbs-Duhem e verificar se os dados experimentais são coerentes e podem ser 
aplicados em algum estudo ou projeto. Ainda na Equação 5, o coeficiente de fugacidade da fase vapor $\left(\phi^{V}\right)$ é calculado através da Equação de Estado (EdE) de Peng-Robinson (Peng e Robinson, 1976). Esta equação é capaz de correlacionar e predizer propriedades de equilíbrio de líquido-vapor.

\section{MODELO TERMODINÂMICO - EQUAÇÃO CÚBICA DE ESTADO DE PENG-ROBINSON}

O método da equação de estado (EdE) é utilizado para correlacionar e predizer o equilíbrio de fases de sistemas complexos. Combinações apropriadas e regras de mistura são utilizadas para descrever a dependência de concentração dos parâmetros do modelo. As equações de estado cúbicas, que são aquelas derivadas da proposta de Van der Waals, tal como a EdE de Peng-Robinson, são muito utilizadas para análise do comportamento termodinâmico destes sistemas complexos (Peng e Robinson, 1976).

A EdE de Peng-Robinson, através de seus parâmetros, regras de mistura e das propriedades críticas dos componentes envolvidos, permite predizer os valores dos coeficientes de fugacidade dos componentes que formam as misturas binárias e ternárias e, assim, aplicar a abordagem gamma-phi a estes sistemas para predição e modelagem dos dados obtidos experimentalmente (Arce, 2003; Valderrama, 2003). Esta equação pode ser representada de duas formas principais: em termos de P, V e T (Equação 6), ou em termos do fator de compressibilidade, z (Equação 7).

$$
P=\frac{R T}{v-b}-\frac{a_{c}}{v(v+b)+b(v-b)}
$$

$$
z^{3}-(1-B) z^{2}+\left(A-2 B-3 B^{2}\right) z-A B+B^{2}+B^{3}=0
$$

Na Equação 6, tem-se que $\mathrm{P}$ é a pressão do sistema, $\mathrm{R}$ é a constante geral dos gases ideais, T é a temperatura absoluta, $v$ é o volume molar, e $a_{c}$, e $b$ são constantes. Essas constantes dependem das propriedades críticas e das regras de misturas. Para determinar o valor do fator de compressibilidade em cada fase, calcula-se o valor do coeficiente de fugacidade correspondente à fase. Neste caso, apenas é de interesse o maior valor de z que corresponde à fase vapor. As constantes " $A$ " e "B" na Equação 7 variam de acordo com a substância e são determinadas usando as Equações 8 e 9 e as regras de mistura (Equações 10, 11 e 12).

$$
\begin{aligned}
& A=\frac{a P}{R^{2} T^{2}} \\
& B=\frac{b P}{R T} \\
& a=\sum_{i}^{C} \sum_{j}^{C} y_{i} y_{j} a_{i j} \\
& a_{i, j}=\left(1-k_{i, j}\right) \sqrt{a_{i} a_{j}}
\end{aligned}
$$




$$
b=\sum_{i}^{C} y_{i} b_{i}
$$

Nos cálculos, o valor de $\mathrm{k}_{\mathrm{ij}}$ foi mantido em zero. Por fim, o modelo termodinâmico propõe que o coeficiente de fugacidade, obtido com a EdE de Peng-Robinson, a ser usado na abordagem gamma-phi pode ser calculado pela Equação 13:

$$
\ln \hat{\phi}_{i}^{V}=(z-1)-\ln (z-B)+\frac{A}{2 \sqrt{2 B}} \ln \left(\frac{z+(1-\sqrt{2}) B}{z+(1+\sqrt{2}) B}\right)
$$

\section{RESULTADOS E DISCUSÕES}

Ao aplicar-se o método de análise de consistência termodinâmica de dados experimentais aos sistemas de baixas pressões mostrados na Tabela 1:

Tabela 1 - Características dos sistemas binários testados com a abordagem gamma-phi.

\begin{tabular}{ccccc}
\hline Sistema & $\begin{array}{c}\text { Pontos } \\
\text { experimentais }\end{array}$ & Temperatura (K) & Pressão (kPa) & Referências \\
\hline terc-Butanol + Tolueno & 26 & $383,8-355,7$ & 101,3 & Martinez-Soria et al. (1999) \\
\hline Propano + n-butano & 16 & $270,0-310,0$ & 142,6 a 720,6 & Kayukawa et al. (2005) \\
\hline Propano + isobutano & 33 & $260,0-320,0$ & 161,2 a 1318,3 & Kayukawa et al. (2005) \\
\hline Pentano + dodecano & 16 & $299,6-453,6$ & 70,0 e 100,0 & Maia de Oliveira et al. (2002) \\
\hline Metanol + benzeno & 10 & $331,1-343,8$ & 101,3 & Rodrigues et al. (2005) \\
\hline Heptano + dodecano & 18 & $345,2-482,1$ & 40,$0 ; 70,0$ e & Maia de Oliveira et al. (2002) \\
& & & 100,0 & \\
\hline Etanol + água & 13 & 333,1 & 48,3 a 72,6 & Mertl (1972) \\
\hline Etano + trifluorometano & 40 & $188,3-212,8$ & 56,9 a 85,4 & Zhang et al. (2006) \\
\hline Dietilamina + etanol & 26 & $303,6-345,6$ & 40,0 e 80,0 & Yang et al. (2014) \\
\hline $\begin{array}{c}\text { Dietilamina + } \\
\text { dietiletanolamina }\end{array}$ & 30 & $303,6-428,1$ & 40,0 e 80,0 & Yang et al. (2014) \\
\hline Cicloexano + n-heptano & 17 & $353,8-371,4$ & 100,7 & Rodrigues et al. (2005) \\
\hline Etanol + dietiletanolamina & 32 & $329,6-428,1$ & 40,0 e 80,0 & Yang et al. (2014) \\
\hline
\end{tabular}

Como explicitado na Equação 3, para serem considerados dados experimentais termodinamicamente consistentes, o valor da integral deve ser igual ou muito próximo de zero. Porém, ao lançar mão de técnicas numéricas de integração, comete-se um erro sistemático da própria técnica e os resultados sofrem leves desvios do valor que seria obtido com a integração pelo método analítico. Sendo assim, considerou-se um desvio de $\pm 0,07$ no valor absoluto obtido para classificar os dados como termodinamicamente consistentes ou não. Os resultados obtidos aplicando-se todos esses critérios encontram-se na Tabela 2. Considerando-se estas condições, obtiveram-se os seguintes resultados finais: os sistemas binários: propano + n-butano, pentano + dodecano, heptano + dodecano, dietilamina + etanol e dietilamina + dietiletanolamina foram considerados termodinamicamente inconsistentes, pois o valor da integral excede a margem de erro considerada; os sistema binários: terc- 
butanol + tolueno, propano + isobutano, metanol + benzeno, etanol + água, etano + trifluorometano, cicloexano + n-heptano e etanol + dietiletanolamina foram considerados termodinamicamente consistentes (o valor da integral dentro da margem de erro considerada).

Tabela 2 - Resultados da consistência termodinâmica.

\begin{tabular}{ccc}
\hline Sistema & Valor da Integral $(*)$ & Consistência termodinâmica \\
\hline Ter-Butanol + Tolueno & 0,00914 & CONSISTENTE \\
\hline Propano + n-Butano & 0,19251 & INCONSISTENTE \\
\hline Propano + Isobutano & 0,03904 & CONSISTENTE \\
\hline Pentano + Dodecano & $-3,70068$ & INCONSISTENTE \\
\hline Metanol + Benzeno & $-0,02811$ & CONSISTENTE \\
\hline Heptano + Dodecano & $-1,09875$ & INCONSISTENTE \\
\hline Etanol + Água & $-0,05315$ & CONSISTENTE \\
\hline Etano + Trifluorometano & $-0,06262$ & CONSISTENTE \\
\hline Dietilamina + Etanol & 0,22373 & INCONSISTENTE \\
\hline Dietilamina + Dietiletanolamina & 0,17233 & INCONSISTENTE \\
\hline Ciclohexano + n-Heptano & $-0,01754$ & CONSISTENTE \\
\hline Etanol + Dietiletanolamina & 0,03568 & CONSISTENTE \\
\hline
\end{tabular}

(*) valores absolutos obtidos aplicando-se a Equação 3 através do método: integração numérica (Simpson).

Isto significa que os dados dos primeiros sistemas não podem ser utilizados para projetos maiores, pois poderiam causar erros de cálculo e, possivelmente, prejuízos ao usuário. Por outro lado, os dados experimentais dos demais sistemas binários são considerados de uso confiável, ou seja, podem ser aplicados em grandes projetos sem o receio de acarretarem prejuízos (Raman, 1985)

\section{CONCLUSÕES}

As conclusões parciais sobre os resultados desta pesquisa são (a) Nem todos os dados experimentais de ELV apresentados na literatura são confiáveis e devem ser submetidos a testes de consistência termodinâmica antes de ganharem utilidade e alguns desses dados experimentais não obedecem à equação de Gibbs-Duhem e não podem ser considerados termodinamicamente consistentes; (b) $\mathrm{O}$ método proposto neste trabalho, a abordagem gamma-phi, é capaz de concluir se os dados experimentais de ELV a baixas pressões de um determinado sistema binário podem ser considerados termodinamicamente consistentes; e (c) A equação de estado de Peng-Robinson precisa apenas de propriedades críticas para obter os coeficientes de fugacidade dos componentes de sistemas binários.

\section{REFERÊNCIAS BIBLIOGRÁFICAS}

ARCE, P.F. Modelagem e Computação do Equilíbrio Multifásico de Fluidos e Fenômenos Críticos em Solubilidades de Polímeros em Misturas de Dióxido de Carbono Supercrítico + Co-solvente. Primeiro Relatório: Doutorado em Engenharia Química. Faculdade de Eng. Química, UNICAMP, SP, Brasil, 2003. 
KAYUKAWA, Y.; FUJII, K.; YUKISHIRO, Y. Vapor-Liquid Equlibrium (VLE) Properties for the Binary Systems Propane (1) + n-Butane (2) and Propane + Isobutane (3). $J$. Chem. Eng. Data, v. 50, p. 579-582, 2005.

LIEBERMANN, E.; FRIED, V. Thermodynamic Consistency Test Methods. Ind. Eng. Chem. Fundam., v.11, p. 280-281, 1972.

MAIA DE OLIVEIRA, H. N.; BEZERRA LOPES, F. W.; DANTAS NETO, A. A.; CHIAVONE-FILHO, O. Vapor-Liquid Equilibria for Pentane + Dodecane and Heptane + Dodecane at Low Pressures. J. Chem. Eng. Data, v. 47, p. 1384-1387, 2002.

MARTINEZ-SORIA, V.; PEÑA, M. P.; MONTON, J. B. Vapor-Liquid Equlibrium for the Binary Systems tert-Butyl Alcohol + Toluene, + Isoctane, and + Methylcyclohexane at 101.3 kPa. J. Chem. Eng. Data, v. 44, p. 148-151, 1999.

McDERMOTT, C.; ELLIS, S.R.M. A Multicomponent Consistency Test. Chem. Eng. Sci., v. 20, p. 293-296, 1965.

MERLT, I. Liquid-vapor equilibrium. Il Phase equilibria in the ternary system ethyl acetate etanol - water. Collect. Czech. Chem. Commun., v. 37, p. 366-374, 1972.

PENG, D.Y.; ROBINSON, D. B. A new two-constant equation of state. Ind. Eng. Chem. Fundam., v. 15, p. 59-64, 1976.

RAMAN, R. Chemical Process Computations. Ed. Elsevier Applied Science Publishers, New York, 1985.

RODRIGUES, W. L.; MATTEDI, S.; ABREU, J. C. N. Experimental Vapor-Liquid Equilibria Data for binary mixtures of xylene isomers. J. Chem. Eng., v. 22, p. 453-652, 2005.

SMITH, J. M.; VAN NESS, H. C.; ABOTT, M. M. Introdução à Termodinâmica da Engenharia Química, 7a Ed., Rio de Janeiro: LTC - Livros Técnicos e Científicos Editora, 2007.

VALDERRAMA, J. O. The state of the cubic equations of state, Ind. Eng. Chem. Research, v. 42, p. 1603-1618, 2003.

YANG, C.; ZHANG, P.; QIN, Z.; FENG, Y.; ZENG, H.; SUN, F. Isobaric Vapor-Liquid Equilibrium for the Binary Systems (Diethylmine + Ethanol), (Ethanol + N,NDiethylethanolamine), and (Diethylamine $+\mathrm{N}, \mathrm{N}-$ Diethylethanolamine $)$ at $\mathrm{p}=(80.0$ and 40.0) kPa. J. Chem. Eng. Data, v. 59, p. 750-756, 2014.

ZHANG, Y.; GONG, M.; ZHU, H.; WU, J. Vapor-Liquid Equilibrium Data for the Ethane + Trifluoromethane System at Temperatures from (188.31 to 243.76) K. J. Chem. Eng. Data, v. 51, p. 1411-1414, 2006. 